\title{
Clinical and Magnifying Narrow-Band Imaging Endoscopic Finding-Olmesartan-Associated Collagenous Gastroduodenitis
}

\author{
Pankaj Desai ${ }^{1} \quad$ Chintan Patel ${ }^{1} \quad$ Mayank Kabrawala ${ }^{2}$ \\ Krishna Parekh ${ }^{4}$ \\ ${ }^{1}$ Department of Endoscopy \& Endosonography, Surat Institute of \\ Digestive Sciences (SIDS), Surat, Gujrat, India \\ ${ }^{2}$ Department of Gastroenterology, Surat Institute of Digestive \\ Sciences (SIDS), Surat, Gujrat, India \\ ${ }^{3}$ Department of pathology, Surat Institute of Digestive Sciences \\ (SIDS), Surat, Gujrat, India \\ ${ }^{4}$ Department of Clinical Research, Surat Institute of Digestive \\ Sciences (SIDS), Surat, Gujrat, India
}

J Digest Endosc 2020;11:146-148
Subhash Nandwani ${ }^{2}$ Priya Arora ${ }^{3}$

\begin{abstract}
Address for correspondence Pankaj Desai, MS, FASGE, FAIGE, Department of Endoscopy \& Endosonography, Surat Institute of Digestive Sciences (SIDS), Majura Gate, Ring Road, Surat 395002, Gujrat India (e-mail: drp_desai@hotmail.com).
\end{abstract}

\begin{abstract}
Keywords

- collagenous gastroduodenitis

- M-NBI finding

- olmesartan

Collagenous gastroduodenitis is a rare and may be associated with olmesartan, an angiotensin-II receptor antagonist used for management of hypertension. It is characterized by marked subepithelial collagen deposition with mucosal inflammatory infiltrate. The characteristic endoscopic finding of collagenous gastritis is nodular mucosa and other findings include erythema friability, erosions, ulcers and atrophy. Olmesartan-associated collagenous gastroduodenitis without colonic involvement is exceptionally rare with only one case reported in literature to date. The association may be difficult to recognize because of its clinical and histologic similarity to the clinical entity collagenous gastroduodenitis. We present a case of Olmesartan-associated collagenous gastroduodenitis, which was observed on magnifying narrow-band imaging endoscopic findings.
\end{abstract}

\section{Introduction}

Collagenous gastroenteritis is relatively uncommon and believed to be a rare clinicopathological condition that involves the gastrointestinal (GI) tract. It is characterized by the subepithelial deposition of collagen bands thicker than $10 \mu \mathrm{m}$, accompanied with the infiltration of inflammatory mononuclear cells in the lamina propria. It includes collagenous colitis, collagenous duodenitis, and collagenous gastritis in descending order of occurrence. The exact etiology and pathogenesis is still in inception, but it is suspected to be immune-mediated and may be associated with certain drugs including olmesartan, an angiotensin-II receptor antagonist. ${ }^{1,2}$

Rubio-Tapia et al was first to observe and report the association of olmesartan with sprue-like enteropathy in 22 patients. Since then, around 100 cases were reported in English literature that support olmesartan-associated enteropathy.
Moreover, several cases reported olmesartan-associated collagenous duodenitis, few on collagenous gastritis, and only rare reports involving both stomach and small bowel. The association may be difficult to recognize because of its clinical and histologic similarity to the other clinical entity. ${ }^{2}$ Here, we present a case of olmesartan-associated collagenous gastroduodenitis that was diagnosed through clinical and magnifying narrow-band imaging (M-NBI) endoscopic findings.

\section{Case Report}

A 54-year-old female presented with postprandial vomiting, unintentional $6 \mathrm{~kg}$ weight loss over 6 months, and diarrhea for the last 2 days. Her medical history included hypertension treated with olmesartan $40 \mathrm{mg}$ once a day for the last 2 years and diabetes mellitus treated with combination of glimepiride and metformin for the last 13 years. Conservative 
treatment of empirical antibiotics did not relieve her symptoms. IgA, t TGA and additional tests were negative for HLA DQ 2/8 gene, which is associated with celiac disease. Hence, she underwent upper GI endoscopy that revealed atrophic mucosa with nodularity in body region of stomach and duodenum ( - Fig. 1a). Endoscopic M-NBI showed tubular structure on the surface of nodules with micromucosal pattern (-Fig. 1b) which was suggestive of collagenous gastroduodenitis. Her colonoscopy showed normal colon, and stool studies to detect infected stool were negative. Histopathological examination of multiple biopsy specimens from stomach and duodenum showed infiltration, with inflammatory cells in lamina propria and severely atrophied epithelium (-Fig. 2a). Masson's trichome staining revealed markedly thickened subepithelial collagen bands ( - Fig. $2 \mathbf{2 b}$ ), which provides definitive diagnosis of collagenous gastroduodenitis. Moreover, her clinical history suggests collagenous gastroduodenitis was associated with olmesartan. The patient was started with amlodipine and olmesartan was discontinued. Follow-up after 12 weeks revealed near-to-complete clinical response and endoscopic M-NBI showed normal micromuco-

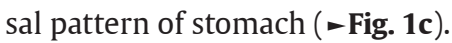

\section{Discussion}

Olmesartan-induced collagenous sprue and gastritis is frequently reported but olmesartan-induced collagenous gastroduodenitis is extremely rare. Our extensive literature search revealed a case study that reported on olmesartan-associated collagenous gastroduodenitis, which highlights bleeding complication while performing endoscopy. We are second to report olmesartan-associated collagenous gastroduodenitis. In the present case, our place of interest is on diagnosis of collagenous gastroduodenitis on $\mathrm{M}-\mathrm{NBI}$ while performing endoscopy.

Olmesartan-associated gastroenteritis is perhaps most familiarly typified by collagenous sprue-like enteropathy. ${ }^{2}$ Graywood et al compiled a 6-year review of pathology reports of patients investigated in the US which showed no association between use of olmesartan and histopathological diagnosis of celiac disease which, in turn, suggests these severe gastrointestinal disorders are a rare adverse effect of this angiotensin II receptor antagonist. ${ }^{3}$ Moreover, Rubio-Tapia et al have already reported that patients with autoimmune disorder have increased prevalence of olmesartan-associated enteropathy.
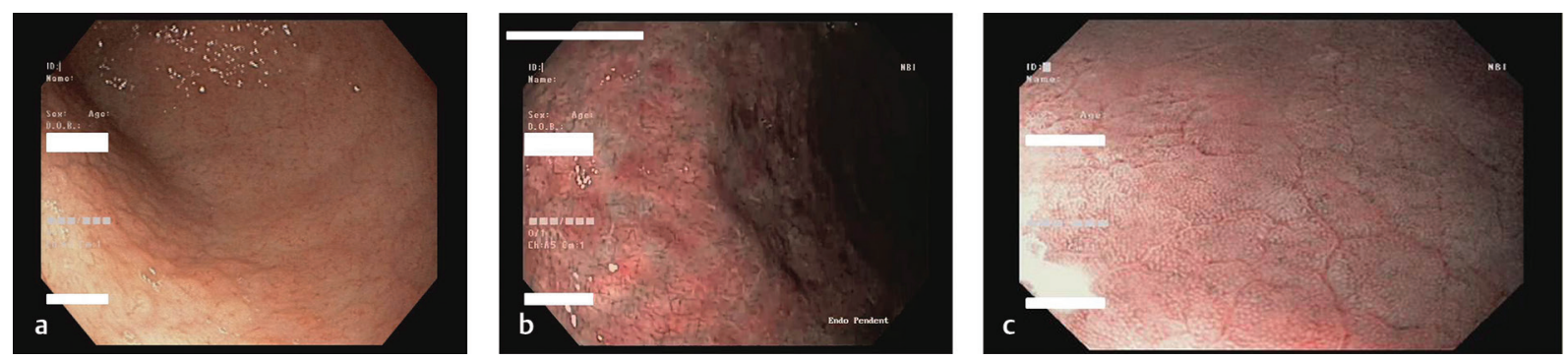

Fig. 1 (a) Endoscopic examination showed nodular gastric and duodenal mucosa. (b) Narrow-band imaging with magnification endoscopy showed tubular structure on the surface of nodules with micromucosal pattern. (c) Narrow-band imaging with magnification endoscopy showed normal micromucosal pattern of stomach.
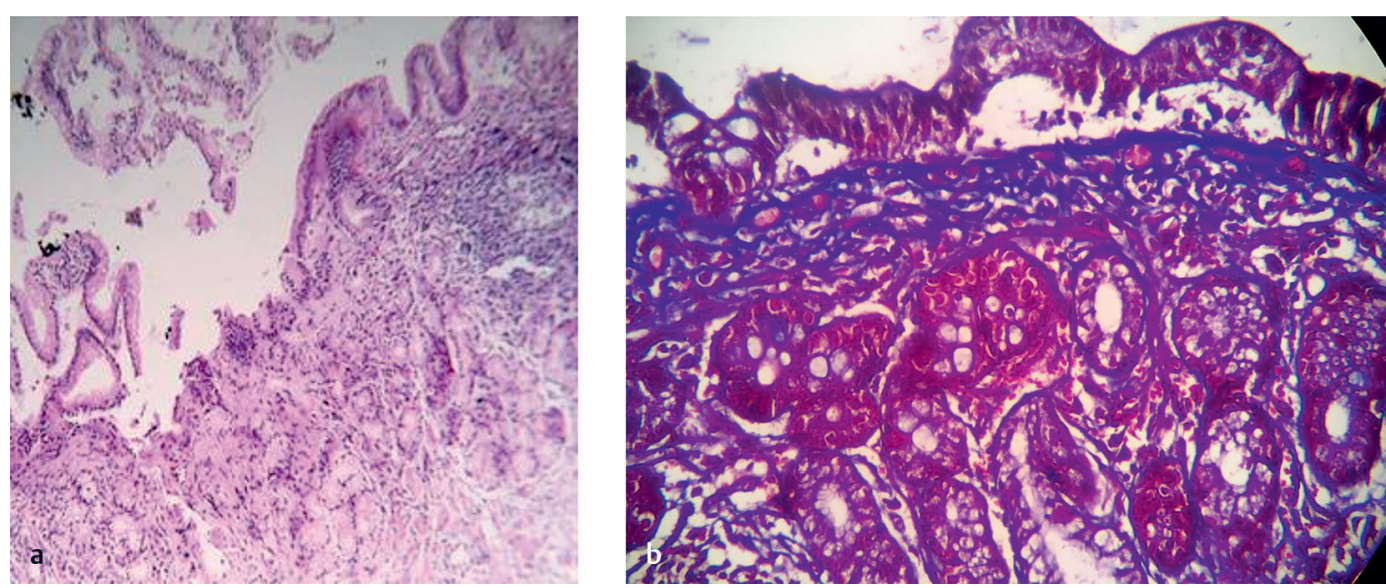

Fig. 2 Histopathological examination of gastric and duodenal biopsies (a) H\&E staining of suggestive of collagenous gastroduodenitis (b). Trichome staining of gastric biopsy showed collagenous gastroduodenitis. Abbreviations: H\&E, hemotoxylin and eosin. 
The mechanism of olmesartan-associated enteropathy is currently unknown. The latency period between initiation of olmesartan and development of symptoms varies from 1 month to 11 years. In our case, the symptoms developed after 2 years of olmesartan. Such a long latency period is suggestive of cell-mediated immune damage. The hypothesis of mechanism in a line is an inhibitory effect of angiotensin II receptor antagonists on transforming growth factor $\beta$ (TGF- $\beta$ ), a multifunctional cytokine. It is a potent immune regulator, which controls synthesis and degradation of extracellular matrices which are necessary for gut homeostasis. Thus, collective inhibition of TGF- $\beta$ leads to inflammatory response and deposition of collagen., ${ }^{2,3}$ Interestingly, the cases due to other angiotensin II receptor antagonists are rarer than olmesartan-associated enteropathy. The selective role of olmesartan might be explained by its conversion into active form in the GI tract, long half-life and 30 folds higher efficacy than other angiotensin-II receptor antagonists.

An endoscopic finding of collagenous gastritis is characterized by fine and fading nodularity surrounding slightly depressed atrophic mucosa. The diagnostic histologic features of collagenous gastritis include dense collagen deposition which is found in the intervening atrophic mucosa, whereas nodules contain inflammatory infiltrates without thickened collagen band. ${ }^{2}$ However, little literature reported on the findings of $\mathrm{M}-\mathrm{NBI}$ for collagenous gastritis.
Kawamura et al reported a tubular gastric micromucosal pattern under $\mathrm{M}-\mathrm{NBI}$ is representative of infiltration of inflammatory cells, and degeneration of surface structure with irregular, narrowed and coiled subepithelial capillaries is compatible with moderate-to-severe mucosal injury and intestinal metaplasia. ${ }^{4}$ Our case suggests that the existence of these distinctive M-NBI findings may be useful for diagnosis of collagenous gastroduodenitis. Thus, we recognize the association of olmesartan with collagenous gastroduodenitis through M-NBI findings and medical history.

\section{Conflicts of Interest}

None declared.

\section{References}

1 Owen DR, Owen DA. Celiac disease and other causes of duodenitis. Arch Pathol Lab Med 2019;2018(139):1242-1247

2 Choi E-YK, McKenna BJ. Olmesartan-associated enteropathy: a review of clinical and histologic findings. Arch Pathol Lab Med 2015;139(10):1242-1247

3 Greywoode R, Braunstein ED, Arguelles-Grande C, Green PH, Lebwohl B. Olmesartan, other antihypertensives, and chronic diarrhea among patients undergoing endoscopic procedures: a case-control study. Mayo Clin Proc 2014;89(9):1239-1243

4 Boeriu A, Boeriu C, Drasovean S, et al. Narrow-band imaging with magnifying endoscopy for the evaluation of gastrointestinal lesions. World J Gastrointest Endosc 2015;7(2):110-120 